\title{
DO THE MIXED CRYSTALS HgSeS EXHIBIT THE ONE-MODE BEHAVIOR?**
}

\author{
W. Szuszkiewicz, A.M. Witowski \\ Institute of Experimental Physics, Warsaw University \\ Hoża 69, 00-681 Warszawa, Poland \\ C. JULien, M. BaLkanski
}

Laboratoire de Physique des Solides, Université Paris VI, France

K. DyBko, B. WitKowska and A. Mycielski

Institute of Physics, Polish Academy of Sciences, Warszawa, Poland

\begin{abstract}
The low-temperature far-infrared reflectivity spectra of $\mathrm{HgSe}$ and $\mathrm{HgSeS}$ are measured and analysed. The results suggest the one-mode behavior of phonon excitations in HgSeS mixed crystals.
\end{abstract}

PACS numbers: 78.20.Ls, 78.30.Fs

The physical properties of the $\mathrm{HgSe}: \mathrm{Fe}$ (and, in particular, of the iron resonant donor state in $\mathrm{HgSe}$ ) were intensively investigated within the last few years. Recently the studies of various kinds of mixed crystals, based on $\mathrm{HgSe}$ and highly doped with iron, have been started. One of the systems, for which the interesting phenomena could be expected, is the HgSeS:Fe mixed crystal. The purpose of this work was to study the far-infrared optical properties of such a system in order to determine the basic properties of the host crystal.

The $\mathrm{HgSeS}: \mathrm{Fe}$ mixed crystals as well as $\beta$ - $\mathrm{HgS}$ have inverted band structure [1] and are always the $n$-type materials. Therefore, the reflectivity measurements are the only way to study the low-energy excitations in these crystals. The phonon spectra for HgSe were analysed in [2] and are relatively well known (the TO-phonon frequency in $\mathrm{HgSe}$ is close to $132 \mathrm{~cm}^{-1}$ ). On the contrary, the phonon spectrum of $\beta-\mathrm{HgS}$ has never been published. The only information about the TO-phonon frequency in this material (being equal to $177 \mathrm{~cm}^{-1}$ ) can be found in [3], where the optical properties of thin $\beta$ - $\mathrm{HgS}$ films on $\mathrm{NaCl}$ substrate were discussed. Also the far-infrared optical properties of the HgSeS mixed crystals have not been studied until now.

*This work is in part supported by the Committee for Scientific Research under grant No. 204759101. 
The low-temperature far-infrared reflectivity spectra of $\mathrm{HgSe}$ and $\mathrm{HgSeS}$ were obtained at $4.5 \mathrm{~K}$ in the spectral range from $100 \mathrm{~cm}^{-1}$ up to $1200 \mathrm{~cm}^{-1}$ using the Fourier Transform Spectrometer. The mechanically polished and chemically etched bulk samples obtained by the Bridgman method were mounted on the cold finger of helium cryostat. The spectral resolution was better than $2 \mathrm{~cm}^{-1}$. The electron concentration for all investigated samples was a few times $10^{18} \mathrm{~cm}^{-3}$, so the plasma edge was always well above the TO-phonon frequency. The experimental curves have been theoretically reproduced using the classical Dynamic Dielectric Function (DDF) in the simplified form:

$$
\varepsilon(\omega)=\varepsilon_{\infty}+\frac{\omega_{\mathrm{p}^{2}}}{\omega(\omega+\mathrm{i} \gamma)}+\frac{F_{\mathrm{TO}} \omega_{\mathrm{TO}}^{2}}{\omega_{\mathrm{TO}}^{2}-\omega^{2}+\mathrm{i} \Gamma \omega},
$$

where $\varepsilon_{\infty}$ corresponds to the high-frequency dielectric constant, $\omega_{\mathrm{TO}}, F_{\mathrm{TO}}$ and $\Gamma$ are the TO-phonon frequency, oscillator strength and damping, respectively, $\omega_{\mathrm{p}}$ is the plasma frequency. In this model for a given sample the plasma damping parameter $\gamma$ has a constant value (energy independent). The comparison of the theoretical curves and the experimental points in the spectral range corresponding to the vicinity of the phonon structure are shown in Fig. 1. Determined from such
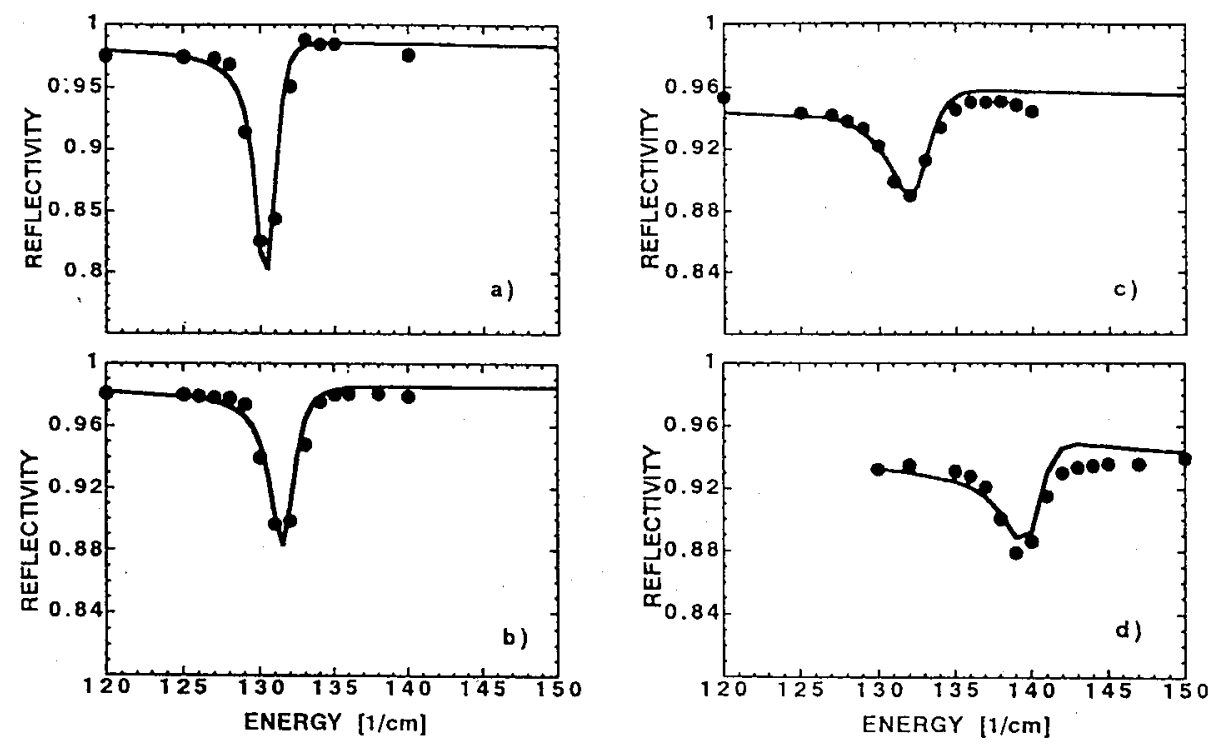

Fig. 1. Part of the $\mathrm{HgSe}_{1-x} \mathrm{~S}_{x}$ reflectivity spectrum in the vicinity of the phonon structure: dots represents the experimental points, solid lines are the theoretical curves calculated from the DDF using Eq. (1) and taking the values of parameters given in Table. a) $\mathrm{HgSe}: \mathrm{Ga}$; b) $\mathrm{HgSe}: \mathrm{Fe}$ c) $\mathrm{HgSe}_{0.8} \mathrm{~S}_{0.2}: \mathrm{Fe}$; d) $\mathrm{HgSe}_{0.4} \mathrm{~S}_{0.6}: \mathrm{Fe}$.

comparisons the best fit values of the parameters: $\omega_{\mathrm{TO}}, F_{\mathrm{TO}}, \Gamma, \omega_{\mathrm{p}}$ and $\gamma$ are given in Table.

When doping $\mathrm{HgSe}$ with $\mathrm{Fe}$ the TO-phonon frequency differs a little from the value corresponding to the "pure" HgSe. However, such changes do not exceed 
$1.0 \mathrm{~cm}^{-1}$ even for the iron concentration as high as $1 \times 10^{20} \mathrm{~cm}^{-1}$, therefore the iron doping of the mixed crystals should not have a great influence on the results obtained. The significant frequency shift of the $\omega_{\text {To }}$ with the increasing sulphur

TABLE

The values of the best fit parameters for the investigated samples.

\begin{tabular}{c|c|c|c|c|c}
\hline \hline Sample & $\begin{array}{c}\omega_{\text {TO }} \\
\left(\mathrm{cm}^{-1}\right)\end{array}$ & $F_{\text {TO }}$ & $\begin{array}{c}\Gamma_{\text {TO }} \\
\left(\mathrm{cm}^{-1}\right)\end{array}$ & $\begin{array}{c}\omega_{\mathrm{p}} \\
\left(\mathrm{cm}^{-1}\right)\end{array}$ & $\begin{array}{c}\gamma \\
\left(\mathrm{cm}^{-1}\right)\end{array}$ \\
\hline $\mathrm{HgSe}: \mathrm{Ga}$ & 131.4 & 5.8 & 0.8 & 667 & 20 \\
$\mathrm{HgSe}: \mathrm{Fe}$ & 132.2 & 5.5 & 1.5 & 750 & 20 \\
$\mathrm{HgSe}_{0.8} \mathrm{~S}_{0.2}: \mathrm{Fe}$ & 133.0 & 3.0 & 2.5 & 750 & 50 \\
$\mathrm{HgSe}_{0.4} \mathrm{~S}_{0.6}: \mathrm{Fe}$ & 140.5 & 3.0 & 2.0 & 890 & 80 \\
$\beta-\mathrm{HgS}$ & $177.0[3]$ & & & &
\end{tabular}

concentration was clearly observed for HgSeS. The dependence of the $\omega_{\text {TO }}$ on the sulphur composition is strongly nonlinear. On the other hand within the experimental error there is no change in the oscillator strength for the TO-phonon when tripling the sulphur concentration from $20 \%$ to $60 \%$ in the HgSeS mixed crystal. No other phonon like structure has been observed. In our opinion these preliminary results strongly suggest a one-mode behavior of the TO-phonon excitation in this material. Most of the mixed crystals exhibit a two-mode behavior. The one-mode behavior was observed for some (not very numerous) mixed crystals (see e.g. [4] and the references), but seems to be the unique case for all known II-VI mixed crystals. It is the open question: is the one-mode behavior typical for all mixed crystals between different mercury chalcogenides or not? More detailed optical studies performed with the higher spectral resolution on the wider group of mixed crystals are necessary to confirm the suggestion about one-mode behavior of $\mathrm{HgSeS}$ and to check the above-mentioned hypothesis.

\section{Acknowledgments}

One of us (W.Sz.) would like to express his gratitude to Laboratoire de Physique des Solides de l'Université Pierre et Marie Curie for the financial support of his stay in Paris.

\section{References}

[1] W. Giriat, Phys. Lett. A 26, 639 (1968); R. Zallen, M. Slade, Solid State Commun. 8, 1291 (1970); K.J. Siemsen, H.D. Riccius, Phys. Status Solidi 37, 445 (1970).

[2] A. Manabe, A. Mitsuishi, Solid State Commun. 16, 734 (1975); A. Manabe, H. Noguchi, A. Mitsuishi, Phys. Status Solidi B 90, 157 (1978); A.M. Witowski, M. Grynberg, Phys. Status Solidi B 100, 389 (1980).

[3] H.D. Riccius, K.J. Siemsen, J. Chem. Phys. 52, 4090 (1970).

[4] G. Lucovsky, M.H. Brodsky, E. Burstein, Phys. Rev. B 2, 3295 (1970). 